\title{
Catheter, surgical, or hybrid procedure: what future for atrial fibrillation ablation?
}

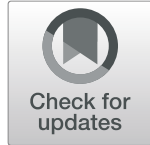

\author{
Giuseppe Nasso ${ }^{1 *}$, Roberto Lorusso ${ }^{2,3}$, Marco Moscarelli', Giuseppe De Martino ${ }^{4}$, Angelo M. Dell'Aquila ${ }^{5}$, \\ Arash Motekallemi ${ }^{5}$, Nicola Di Bari ${ }^{6}$, Ignazio Condello ${ }^{1}$, Pasquale Mastroroberto ${ }^{7}$, Giuseppe Santarpino ${ }^{1,7,8+}$ (iD and \\ Giuseppe Speziale ${ }^{1+}$
}

\begin{abstract}
Background: The debate on the best treatment strategy for atrial fibrillation (AF) has expanded following the introduction of the so-called "hybrid procedure" that combines minimally invasive epicardial ablation with endocardial catheter ablation. However, the advantage of the hybrid approach over conventional epicardial ablation remains to be established.
\end{abstract}

Methods: From June 2008 to December 2020, 609 surgical AF ablation procedures through a right minithoracotomy were performed at our institution. From 2008 to 2011, a unipolar radiofrequency (RF) device was used, whereas from 2011 to 2020 a bipolar RF device was used. In addition, between September 2016 and April 2017, 60 patients underwent endocardial completion of epicardial linear ablation. In 30 of these latter patients, surgical isolation of the Bachmann's bundle (BB) was also performed. Starting from 2021, surviving patients at follow-up were asked to undergo electrocardiographic evaluation and left ventricular function assessment and to complete a questionnaire addressing quality of life and predisposing factors for recurrent AF.

Results: The ablation procedure was completed in all patients. Upon discharge, 30 (4.9\%) patients showed recurrence of AF, whereas the remaining patients (95.1\%) were in sinus rhythm. All patients in whom a hybrid approach was used either with or without BB ablation were discharged in sinus rhythm. After a mean follow-up of 74 months, 122 (20\%) patients developed recurrent AF, including 19.9\% in whom a unipolar RF device was used, $21 \%$ in whom a bipolar RF device was used, $23 \%$ who had undergone a hybrid procedure without BB ablation and $3.3 \%$ who had undergone a hybrid procedure with BB ablation. On multivariate analysis, reduced left ventricular ejection fraction, worsening of European Heart Rhythm Association symptom class, and cognitive impairment or depression during follow-up were found to be significantly associated with AF recurrence.

Conclusions: Surgical AF ablation through a right minithoracotomy is safe and may allow the creation of additional linear lesions, particularly in the BB. The placement of adjunctive linear lesions in the setting of a hybrid procedure can be more effective in reducing the risk for AF recurrence than isolated surgical ablation or hybrid ablation without the addition of further linear lesions, with no incremental risk to the patient.

Keywords: Surgical ablation of atrial fibrillation, Catheter ablation of atrial fibrillation, Hybrid ablation of atrial fibrillation

\footnotetext{
* Correspondence: gnasso@gvmnet.it

${ }^{\dagger}$ Giuseppe Santarpino and Giuseppe Speziale are contributed equally as senior authors.

'Department of Cardiac Surgery, GVM Care\&Research, Anthea Hospital, Via Camillo Rosalba, 35, /38 Bari, Italy

Full list of author information is available at the end of the article
}

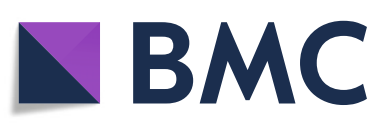

(c) The Author(s). 2021 Open Access This article is licensed under a Creative Commons Attribution 4.0 International License, which permits use, sharing, adaptation, distribution and reproduction in any medium or format, as long as you give appropriate credit to the original author(s) and the source, provide a link to the Creative Commons licence, and indicate if changes were made. The images or other third party material in this article are included in the article's Creative Commons licence, unless indicated otherwise in a credit line to the material. If material is not included in the article's Creative Commons licence and your intended use is not permitted by statutory regulation or exceeds the permitted use, you will need to obtain permission directly from the copyright holder. To view a copy of this licence, visit http://creativecommons.org/licenses/by/4.0/. The Creative Commons Public Domain Dedication waiver (http://creativecommons.org/publicdomain/zero/1.0/) applies to the data made available in this article, unless otherwise stated in a credit line to the data. 


\section{Background}

Prevalence of atrial fibrillation (AF) is increasing worldwide and its management poses challenges that prompted the recent update of the European Society of Cardiology/European Association of CardioThoracic Surgery guidelines [1]. Catheter ablation of AF improved significantly over the past decade, but many patients still experience recurrent AF after the procedure leading to the need for repeat ablation [2, 3]. In current guidelines, surgical ablation of AF is not recommended as first-line therapy due to the limited, though promising, available evidence [4-6], but it may be considered as initial therapy in selected patients [1]. In particular, by comparing guideline recommendations of 2016 vs 2020, catheter or surgical ablation should be considered in patients with symptomatic persistent or long-standing persistent AF (Class IIb in 2016), but only AF catheter ablation for pulmonary vein isolation is recommended for rhythm control after one failed or intolerant drug therapy (Class I in 2020) [1]. Thoracoscopic or hybrid surgical ablation for patients refractory to drug therapy or after failed percutaneous AF ablation stays as a Class IIa recommendation [1].

Recently, it has been shown that electrical isolation of the left atrial posterior wall, particularly when targeting the Bachmann's bundle (BB), may play a key role in ensuring procedural success [7]. BB may be involved in the pathogenesis of AF by sustaining a number of unstable reentrant circuits, and $\mathrm{BB}$ isolation has been hypothesized to prevent induction of stable AF [7-10]. As indicated by current guidelines, the achievement of complete ablation is of great relevance. The aim of this study was to evaluate the efficacy of endoscopic ablation, with and without the creation of adjunctive linear lesions at the $\mathrm{BB}$ region, in preventing or detecting gaps in ablation lines in patients who have previously undergone epicardial ablation, compared to isolated surgical ablation.

\section{Methods}

From June 2008 to December 2020, 609 consecutive patients underwent surgical or two-staged hybrid ablation at the Anthea Hospital, Gruppo Villa Maria (GVM) Care \& Research, Bari, Italy, and were followed up to monitor AF recurrences. Surgical treatment of AF was performed through a right minithoracotomy using a unipolar radiofrequency (RF) device from 2008 to 2011 (Estech, Cobra Adhere XL) and a bipolar RF device (i.e. unidirectional device with two electrodes) from 2011 onward (Estech COBRA Fusion $^{\mathrm{TM}} 150$ Surgical Ablation System). All enrolled patients underwent epicardial isolation of the pulmonary veins (PVs) and the left atrial posterior wall ("box lesion").
Our operative technique has been described elsewhere [4]. Briefly, a 3 to $4 \mathrm{~cm}$ right minithoracotomy was performed at the level of the 3rd intercostal space. The devices deliver bipolar or unipolar RF energy with the aim to obtain electrical PV isolation by temperature controlled RF ablation of the atrial myocardium. The ablation was performed by two energy applications lasting $150 \mathrm{~s}$ each. These were followed by a $60 \mathrm{~s}$ application after the probe was moved circumferentially, to achieve complete closure of the box lesion. All procedures were performed off-pump. A circular box lesion was created.

Among hybrid patients $(n=60), 30$ patients underwent surgical ablation with additional linear lesions targeting the $\mathrm{BB}$. The $\mathrm{BB}$ was ablated by introducing the magnetic tip below the ascending aorta and above the roof of the left atrium and then advanced to the base of the left atrial appendage. No further dissection was required.

As previously described [7], hybrid patients underwent a staged endocardial ablation within 6 weeks after the surgical procedure with the aim at verifying or ablating: (i) the surgical lines and completing isolation of the box lesion if needed, (ii) additional right and left atrial substrate modification, and (iii) other triggers of AF.

Catheter ablation was performed under general anesthesia and esophageal temperature monitoring with a dedicated tripolar catheter (Esotherm, Fiab). Mapping and ablation were performed using an electroanatomic mapping system (CARTO, Biosense Webster, Diamond Bar, CA, USA). A detailed bipolar voltage map of the left atrium was obtained. All points were acquired point-bypoint using the ablation catheter to ensure adequate catheter tissue contact by contact force. RF was applied using an open irrigated tip catheter with power output up on the posterior wall and in the remaining atrial sites. Entrance block was defined by complete elimination or dissociation of PV potentials, determined by the circular mapping catheter positioned in the PVs and posterior wall [7]. Endocardial ablation was first directed to possible gaps in the surgical lesions. Moreover, the procedure was completed with:

- ablation of the Marshall ligament,

- roof and anterior mitral lines,

- coronary sinus and superior vena cava isolation,

- intercaval and cavotricuspid isthmus lines.

Patients were then referred to the local cardiologist with recommended follow-up at 6, 9, 12 months and then every 6 or 12 months depending on rhythm stability. Moreover, starting from 2021, surviving patients were asked to undergo assessment of left ventricular function and to complete a questionnaire addressing their behavior in relation to predisposing factors for recurrent AF, including alcohol and caffeine intake, 
smoking, and weight excess or loss. In addition, patients with diabetes, hypertension or obstructive sleep apnea requiring continuous positive airway pressure were asked if they were receiving appropriate treatment for their disease and were facing any difficulties in compliance and achieving optimal medical therapy. Changes in patients' quality of life were also investigated based on New York Heart Association (NYHA) functional classification and European Heart Rhythm.

\section{Association (EHRA) symptom scale}

In particular, the questions for the patients in the interview included the following:

- Do you have dyspnea/respiratory impairment? Can you take the stairs? How many floors?

- Are your symptoms restricting your normal daily activities? Did you have to reduce or stop your normal daily activities?

- Do you smoke or are you continuing to smoke?

- Do you drink alcohol, coffee, tea or take other stimulants?

- Have you lost or gained weight in the past few years?

The risk for recurrent AF was determined by evaluating clinical and echocardiographic parameters recorded at last follow-up on the basis of patient-reported outcomes and questionnaire assessment on postoperative behavioral modifications aimed at reducing triggering factors for AF (i.e. weight excess or loss, reduction or elimination of alcohol and caffeine intake, smoking cessation, optimal management of diabetes or hypertension or sleep apneas). Quality of life was evaluated using NYHA functional classification and the EHRA score of AF-related symptoms.

The GVM Care\&Research ethics committee approved the study and all patients provided written informed consent for the procedure and study enrollment.

\section{Statistical analysis}

Data analysis was performed using Excel 2016 (Microsoft, Redmond, WA, USA) and statistical analysis was performed using SPSS (IBM SPSS Statistics for Windows, Version 27.0. Armonk, NY: IBM Corp). Categorical variables are given as counts and percentages. Event-free estimate such as recurrence of AF was determined using the Kaplan-Meier method. Possible risk factors for AF recurrence are reported in Table 1 and were used for determining the predictive model. To this purpose, univariate analysis was performed first. Variables with a $p$-value $=0.2$ were included in a multivariable model for Cox regression analysis with stepwise selection to determine the independent predictors of $\mathrm{AF}$ recurrence. A p-value $p<0.05$ was considered statistically significant.

\section{Results}

Surgical ablation was completed in all patients and was performed in 324 (53.2\%) patients with paroxysmal AF and $285(46.8 \%)$ patients with persistent AF. Mean age of the study population was 63 (range 27-87) years. The AF ablation procedure was performed using a unipolar RF device in 151 (24.8\%) patients and a bipolar RF device in 398 (65.2\%) patients. A hybrid approach using a bipolar RF device was adopted in 60 patients with additional linear lesions targeting the BB in $30(5 \%)$ patients.

Intraoperative and postoperative complications were recorded in 7 (1.1\%) and $11(1.8 \%)$ patients, respectively. The 30 -day mortality was $0 \%$.

At discharge, most patients (95.1\%) were in sinus rhythm whereas $4.9 \%(n=30)$ were in AF. No patient required pacemaker implantation. Notably, all patients who had undergone hybrid ablation either with or without $\mathrm{BB}$ ablation were discharged in sinus rhythm after completion of the second procedure.

At a mean follow-up of 74 (2-152) months, $4(0.6 \%)$ patients died of non-cardiac causes and $122(20 \%)$ patients experienced recurrent AF. AF recurrence rates by type of ablation procedure are shown in Fig. 1 [unipolar RF ablation: $19.9 \%(n=30)$; bipolar RF ablation: $21 \%$ $(n=84$; hybrid procedure without adjunctive BB ablation: $23 \%(n=7)$; hybrid procedure with adjunctive $\mathrm{BB}$ ablation: $3.3 \%(n=1)]$.

On univariate analysis, intraoperative complications, persistence of AF triggers (overweight, continued alcohol and caffeine consumption), suboptimal management of continuous positive air pressure and diabetes, reduced left ventricular ejection fraction, higher NYHA class, hospitalization due to cardiac causes, the occurrence of cerebrovascular events and cognitive impairment or depression were found to be associated with recurrent $\mathrm{AF}$ during follow-up (Table 1). On multivariate analysis, intraoperative complications, impaired left ventricular ejection fraction, worsening of EHRA symptom class and cognitive impairment or depression during followup remained significantly associated with AF recurrence (Table 1).

\section{Discussion}

Monitoring of patients undergoing surgical or endovascular ablation of AF has evolved remarkably over the last years. Current guidelines emphasize the importance of achieving complete electrical PV isolation [1], a concept that cannot be taken for granted and should not be neglected given that PV reconnection rates are as high as $70 \%$ [1]. 
Table 1 Univariate and multivariate analysis

\begin{tabular}{|c|c|c|c|}
\hline & \multicolumn{2}{|c|}{ Including Bachmann bundle } & \multirow{2}{*}{$\begin{array}{l}\text { Exp } \\
\text { (B)/ } \\
\text { Hazard }\end{array}$} \\
\hline & $\begin{array}{l}\text { Univariate } \\
p \text {-value }\end{array}$ & $\begin{array}{l}\text { Multivariate } \\
\text { p-value }\end{array}$ & \\
\hline Age & 0.426 & & \\
\hline Type of ablation & 0.694 & & \\
\hline Complications intraop & 0.11 & 0.015 & \\
\hline Complications postop & 0.056 & 0.929 & \\
\hline Rhythm & 0.352 & & \\
\hline Rhythm at discharge & 0.834 & & \\
\hline \multicolumn{4}{|l|}{ Follow-up } \\
\hline Exitus & n.a. & & \\
\hline Date & n.a. & & \\
\hline Months & n.a. & & \\
\hline Years & n.a. & & \\
\hline Rhythm & n.a. & & \\
\hline Ejection fraction & 0.453 & & \\
\hline Ejection fraction at follow-up & 0.000 & 0.002 & 0.939 \\
\hline NYHA class & 0.000 & 0.637 & 0.848 \\
\hline Gained $10 \mathrm{~kg}$ & 0.049 & 0.851 & \\
\hline Continued smoking & 0.141 & 0.348 & 0.189 \\
\hline Continued drinking & 0.000 & 0.155 & 0.970 \\
\hline CPAP at follow-up & 0.015 & 0.714 & 0.700 \\
\hline Hypertension at follow-up & 0.319 & & \\
\hline Diabetes at follow-up & 0.054 & 0.327 & \\
\hline Caffeine consumption & 0.001 & 0.610 & 0.733 \\
\hline EHRA score & 0.065 & 0.048 & 0.873 \\
\hline Hospitalization for cardiac causes & 0.000 & 0.305 & 0.941 \\
\hline Cerebrovascular event at follow-up & 0.025 & 0.235 & 1.021 \\
\hline Cognitive impairment or depression & 0.000 & 0.401 & 0.515 \\
\hline \multicolumn{4}{|l|}{ Cox Regression - Method } \\
\hline Ejection fraction at follow-up & 14.099 & 0.000 & \\
\hline EHRA score & 8.641 & 0.003 & \\
\hline Cognitive impairment or depression & 1.275 & 0.259 & \\
\hline
\end{tabular}

CPAP continuous positive airway pressure, EHRA European Heart Rhythm Association, NYHA New York Heart Association

However, despite the large number of catheter ablation procedures, only few patients undergo multidisciplinary heart team discussion for proper decision making about hybrid AF ablation. Although various ablation strategies have been proposed and implemented into clinical practice [11-15], the success rate of catheter ablation in AF patients remains low, with wide variations in ablation techniques among operators. In our study, surgical AF ablation was unsuccessful in one fifth of patients likely due to the lack of mapping and catheter ablation. Increasing evidence suggests that the hybrid approach could represent a more aggressive, but very effective treatment for such patients [16].
Given the not negligible proportion of patients experiencing failed ablations with subsequent poorer long-term clinical outcomes and quality of life, it can be speculated that hybrid surgical-catheter ablation procedures combining a minimally invasive epicardial ablation with no sternotomy and cardiopulmonary bypass with a percutaneous endocardial approach may result in improved outcomes than either procedure alone [10]. However, in our study, although all patients who had undergone two-staged hybrid ablation were discharged in sinus rhythm, we could not demonstrate the superiority of the hybrid procedure over isolated surgical ablation. 


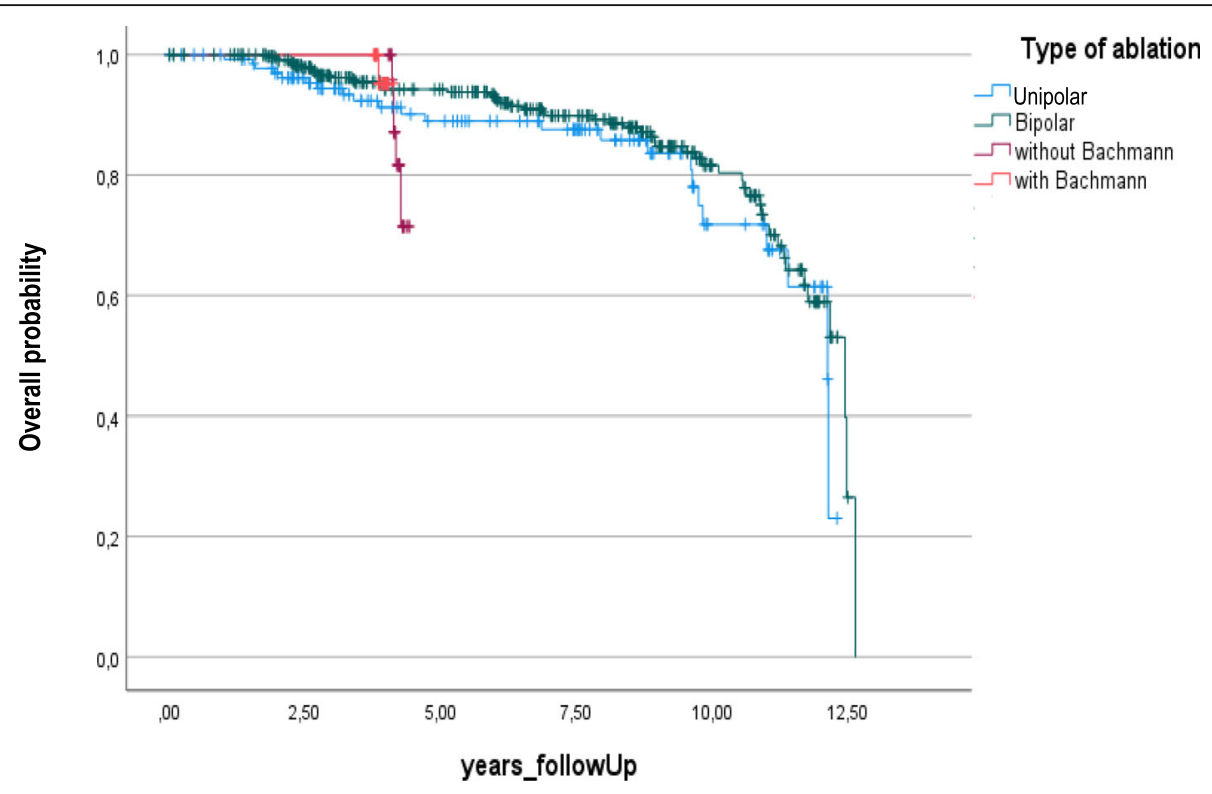

Fig. 1 Atrial fibrillation recurrence by type of ablation procedure. Recurrence rates were 19.9\% with unipolar RF ablation, 21\% with bipolar RF ablation, 23\% with hybrid procedure without adjunctive BB ablation, and 3.3\% with hybrid procedure with adjunctive BB ablation

The main finding of our study was a significant reduction of AF recurrence in hybrid patients in whom adjunctive $\mathrm{BB}$ ablation was performed. In this respect, a few considerations are relevant: (i) BB ablation in the setting of a two-staged hybrid procedure is safe and highly effective; (ii) adding this surgical ablation target, where the $\mathrm{BB}$ is supposed to be anatomically located, was easy to perform without a significant increase in procedural time and without requiring further blunt dissection; and (iii) $\mathrm{BB}$ ablation does not increase the risk for periprocedural complications.

The results obtained were better than those recorded in other centers with hybrid procedures not targeting the $\mathrm{BB}[17-20]$. This could be due to the fact that the $\mathrm{BB}$ may be involved in a number of unstable reentrant circuits, and we hypothesized that an effective lesion in the $\mathrm{BB}$ would prevent induction and maintenance of AF.

It is worth noting that current guidelines also suggest that catheter ablation should be reserved for patients with AF which remains symptomatic despite optimal medical therapy [1]. Besides the clear indication for the need of providing practitioners and institutions with tools to measure the quality of care that AF patients receive so as to identify opportunities for improvement, the impact of lesion sets in addition to PV isolation is still uncertain. The debate remains therefore open and guidelines prompt us to improve the quality of our treatment strategies. Our study contributes in that direction by performing adjunctive $\mathrm{BB}$ ablation with the aim to improve the outcome. Adjunctive BB ablation in the setting of a hybrid surgical approach using minithoracotomy was safe, with an intraoperative complication rate similar to hybrid surgical ablation not targeting the $\mathrm{BB}$ ( $1 \%$ vs $3 \%, p=0.42$ ).

Prospective, registry-based data show that approximately 4 to $14 \%$ of patients undergoing catheter AF ablation experience complications, which means that these data do not differ from those reported with thoracoscopic surgical ablation [1]. Our results show that the rate of intraoperative complications, either with or without adjunctive BB ablation, is similar to that observed with the endoscopic or thoracoscopic approach but such complications can be safely managed through a right minithoracotomy performed under direct vision. Despite being considered more invasive and burdened by higher risk, minimally invasive surgical ablation through a right minithoracotomy can also allow to address technical challenges when performing additional lesion lines (e.g. adjunctive BB ablation [9]), which seem to confer encouraging results but are not considered yet in current guidelines due to the lack of sufficient evidence.

In addition to safety aspects, a few considerations on efficacy deserve mentioning. The FAST trial randomized patients who were prone to AF catheter-ablation failure (i.e. failed previous ablation or left atrial dilation and hypertension) and reported common but substantially lower AF recurrence rates after thoracoscopic compared with catheter ablation ( $56 \%$ vs. $87 \%)$ at long-term follow-up [21]. In our study, among the 30 patients who had undergone adjunctive BB ablation, only one (3\%) had recurrent $\mathrm{AF}$ at a mean follow-up of 47 (45-49) months.

Our study has limitations that include a small sample size and the single-center experience. However, this is 
the largest case series to date of patients treated by minithoracotomy and, associated in some cases with a hybrid approach with or without BB ablation. Further multicenter and randomized experiences are needed to confirm our results.

In conclusion, we believe that both isolated surgical or catheter ablation of AF are destined to provide unsatisfactory results but, at present, only few data are available for the hybrid approach. It would be interesting to understand why the high number of catheter ablation procedures is not counterbalanced by a proportionate number of hybrid procedures.

\section{Conclusions}

The results of our study show that surgical AF ablation through a right minithoracotomy is safe and may be safer than thoracoscopic or catheter ablation according to available evidence. Higher procedural success rates could be obtained from the adoption of a hybrid approach to achieve complete closure of the box lesion with adjunctive $\mathrm{BB}$ ablation.

\section{Abbreviations}

AF: Atrial fibrillation; BB: Bachmann's bundle; EHRA: European Heart Rhythm Association; GVM: Gruppo Villa Maria; NYHA: New York Heart Association; PV: Pulmonary vein; RF: Radiofrequency

\section{Acknowledgements}

Not applicable.

\section{Authors' contributions}

$\mathrm{G}$ Nasso ideation, database, revision, $\mathrm{R}$ Lorusso supervision, M Moscarelli supervision, G De Martino database, AM Dell'Aquila statistic, A Motekallemi statistic, N Di Bari revision, I Condello revision, P Mastroroberto supervision, G Santarpino writing, G Speziale supervision. The authors read and approved the final manuscript.

\section{Funding}

None.

\section{Availability of data and materials}

The datasets used and analysed during the current study are available from the corresponding author on reasonable request.

\section{Declarations}

Ethics approval and consent to participate

obtain, internal ethic board.

\section{Consent for publication \\ Not applicable.}

\section{Competing interests}

None.

\section{Author details}

'Department of Cardiac Surgery, GVM Care\&Research, Anthea Hospital, Via Camillo Rosalba, 35, /38 Bari, Italy. ' Cardio-Thoracic Surgery Department, Heart \& Vascular Centre, Maastricht University Medical Centre (MUMC), Maastricht, Netherlands. ${ }^{3}$ Cardiovascular Research Institute Maastricht (CARI M), Maastricht, Netherlands. ${ }^{4}$ Department of Cardiology and Electrophysiology, Clinica Mediterranea, Naples, Italy. ${ }^{5}$ Department of Cardiac Surgery, Münster Universität, Münster, Germany. ${ }^{6}$ Department of Cardiac Surgery, "Aldo Moro" University, Bari, Italy. ${ }^{7}$ Department of Experimental and
Clinical Medicine, Magna Graecia University, Catanzaro, Italy. ${ }^{8}$ Department of Cardiac Surgery, Paracelsus Medical University, Nuremberg, Germany.

Received: 24 May 2021 Accepted: 14 June 2021

Published online: 26 June 2021

References

1. Hindricks G, Potpara T, Dagres N, Arbelo E, Bax JJ, Blomström-Lundqvist C, et al. 2020 ESC guidelines for the diagnosis and management of atrial fibrillation developed in collaboration with the European Association for Cardio-Thoracic Surgery (EACTS). Eur Heart J. 2020:42:373-498.

2. Muneretto C, Bisleri G, Rosati F, Krakor R, Giroletti L, Di Bacco L, et al. European prospective multicentre study of hybrid thoracoscopic and transcatheter ablation of persistent atrial fibrillation: the HISTORIC-AF trial. Eur J Cardiothorac Surg. 2017;52(4):740-5. https://doi.org/10.1093/ejcts/ezx1 62.

3. Mohanty S, Di Biase L, Mohanty P, Trivedi C, Santangeli P, Bai R, et al. Effect of periprocedural amiodarone on procedure outcome in patients with longstanding persistent atrial fibrillation undergoing extended pulmonary vein antrum isolation: results from a randomized study (SPECULATE). Heart Rhythm. 2015;12(3):477-83. https://doi.org/10.1016/j.hrthm.2014.11.016.

4. Nasso G, MoscarelliM FK, Zebele C, lacopino S, Fiore F, et al. Mid-term performance of bipolar radiofrequency ablation for isolated atrial fibrillation through a right minithoracotomy. Semin Thorac Cardiovasc Surg. 2017;29(2): 160-72. https://doi.org/10.1053/j.semtcvs.2017.01.005.

5. Muneretto C, Bisleri G, Bontempi L, Cheema FH, Curnis A. Successful treatment of lone persistent atrial fibrillation by means of a hybrid thoracoscopic-transcatheter approach. Innovations (Phila). 2012;7(4):254-8. https://doi.org/10.1097/imi.0b013e31826f0462.

6. Muneretto C, Bisleri G, Bontempi L, Curnis A. Durable staged hybrid ablation with thoracoscopic and percutaneous approach for treatment of longstanding atrial fibrillation: a 30-month assessment with continuous monitoring. J Thorac Cardiovasc Surg. 2012;144(6):1460-5. https://doi.org/1 0.1016/j.jtcvs.2012.08.069

7. De Martino G, Nasso G, Gasperetti A, Moscarelli M, Mancusi C, Della Ratta G, et al. Targeting Bachmann's bundle in hybrid ablation for long-standing persistent atrial fibrillation: a proof of concept study. J Interv Card Electrophysiol. 2021;8. https://doi.org/10.1007/s10840-021-00971-7 [Epub ahead of print].

8. Van Campenhout MJH, Yaksh A, Kik C, De Jaegere PP, Ho SY, Allessie MA, et al. Bachmann's bundle a key player in the development of atrial fibrillation? Circ Arrhythm Electrophysiol. 2013;6(5):1041-6. https://doi.org/1 0.1161/CIRCEP.113.000758.

9. Kumagai K, Khrestian C, Waldo AL. Simultaneous multisite mapping studies during induced atrial fibrillation in the sterile pericarditis model: insights into the mechanism of its maintenance. Circulation. 1997;95(2):511-21. https://doi.org/10.1161/01.CIR.95.2.511.

10. Kumagai K, Uno K, Khrestian C, Waldo AL. Single site radiofrequency catheter ablation of atrial fibrillation: studies guided by simultaneous multisite mapping in the canine sterile pericarditis model. J Am Coll Cardiol. 2000;36(3):917-23. https://doi.org/10.1016/S0735-1097(00)00803-2.

11. Dewire J, Calkins $\mathrm{H}$. Update on atrial fibrillation catheter ablation technologies and techniques. Nat Rev Cardiol. 2013;10(10):599-612. https:// doi.org/10.1038/nrcardio.2013.121.

12. Forleo GB, De Martino G, Mantica M, Carreras G, Parisi Q, Zingarini G, et al. Clinical impact of catheter ablation in patients with asymptomatic atrial fibrillation: the IRON-AF (Italian registry on NavX atrial fibrillation ablation procedures) study. Int J Cardiol. 2013;168(4):3968-70. https://doi.org/10.101 6/j.ijcard.2013.06.132.

13. Dukkipati SR, Neuzil P, Kautzner J, Petru J, Wichterle D, Skoda J, et al. The durability of pulmonary vein isolation using the visually guided laser balloon catheter: multicenter results of pulmonary vein remapping studies. Heart Rhythm. 2012;9(6):919-25. https://doi.org/10.1016/j.hrthm.2012.01.019.

14. Kuck KH, Brugada J, Fürnkranz A, Metzner A, Ouyang F, Chun KR, et al. Cryoballoon or radiofrequency ablation for paroxysmal atrial fibrillation. $\mathrm{N}$ Engl J Med. 2016;374(23):2235-45. https://doi.org/10.1056/NEJMoa1602014.

15. Cox JL, Churyla A, Malaisrie SC, Pham DT, Kruse J, Kislitsina ON, et al. A hybrid maze procedure for long-standing persistent atrial fibrillation. Ann Thorac Surg. 2019;107(2):610-8. https://doi.org/10.1016/j.athoracsur.2018.06. 064. 
16. Marchlinski F, Kumareswaran R. Hybrid ablation for atrial fibrillation: better or just different? JACC Clin Electrophysiol. 2017;3(4):350-2. https://doi.org/1 0.1016/j.jacep.2016.12.020.

17. Bisleri G, Rosati F, Bontempi L, Curnis A, Muneretto C. Hybrid approach for the treatment of long-standing persistent atrial fibrillation: electrophysiological findings and clinical results. Eur J Cardiothorac Surg. 2013;44(5):919-23. https://doi.org/10.1093/ejcts/ezt115.

18. Kress DC, Erickson L, Choudhuri I, Zilinski J, Mengesha T, Krum D, et al. Comparative effectiveness of hybrid ablation versus endocardial catheter ablation alone in patients with persistent atrial fibrillation. JACC Clin Electrophysiol. 2017;3(4):341-9. https://doi.org/10.1016/j.jacep.2016.10.010.

19. Budera P, Osmancik P, Herman D, Zdarska J, Talavera D, Mala A, et al. Midterm outcomes of two-staged hybrid ablation of persistent and longstanding persistent atrial fibrillation using the versapolar epicardial surgical device and subsequent catheter ablation. J Interv Card Electrophysiol. 2017; 50(2):187-94. https://doi.org/10.1007/s10840-017-0286-y.

20. Toplisek J, Pernat A, Ruzic N, Robic B, Sinkovec M, Cvijic M, et al. Improvement of atrial and ventricular remodeling with low atrial fibrillation burden after hybrid ablation of persistent atrial fibrillation. Pacing Clin Electrophysiol. 2016;39(3):216-24. https://doi.org/10.1111/pace.12791.

21. Castella M, Kotecha D, van Laar C, Wintgens L, Castillo Y, Kelder J, et al. Thoracoscopic vs catheter ablation for atrial fibrillation: long-term follow-up of the FAST randomized trial. Europace. 2019;21(5):746-53. https://doi.org/1 0.1093/europace/euy325.

\section{Publisher's Note}

Springer Nature remains neutral with regard to jurisdictional claims in published maps and institutional affiliations.

Ready to submit your research? Choose BMC and benefit from:

- fast, convenient online submission

- thorough peer review by experienced researchers in your field

- rapid publication on acceptance

- support for research data, including large and complex data types

- gold Open Access which fosters wider collaboration and increased citations

- maximum visibility for your research: over $100 \mathrm{M}$ website views per year

At $\mathrm{BMC}$, research is always in progress.

Learn more biomedcentral.com/submissions 